\title{
Lanthanide-based luminescent molecular thermometers
}

\author{
Carlos D. S. Brites, ${ }^{a}$ Patricia P. Lima, ${ }^{a}$ Nuno J. O. Silva, ${ }^{a}$ Angel Millán, ${ }^{b}$ Vitor S. Amaral, ${ }^{a}$ Fernando \\ Palacio, ${ }^{b}$ and Luís D. Carlos, ${ }^{a}$
}

\author{
Received (in XXX, XXX) Xth XXXXXXXXX 200X, Accepted Xth XXXXXXXXX 200X \\ ${ }_{5}$ First published on the web Xth $X X X X X X X X X 200 X$ \\ DOI: $10.1039 / b 000000 x$
}

Non-invasive accuarate thermometers with high spatial resolution and operating at sub-micron scales, where the conventional methods are ineffective, is currently a very active field of research strongly stimulated in the last couple of years by the challenging demands of nanotechnology and

10 biomedicine. This review offers a general overview of recent examples of accurate luminescent thermometers working at micrometric and nanometric scales, particularly those involving advanced $\mathrm{Ln}^{3+}$-based functional organic-inorganic hybrid materials.

\section{Introduction}

Sensing and mapping temperature in an accurate and non15 invasive way with submicrometric resolution is critical to understand numerous features of micro and nanoscale electronic and photonic devices, such as thermal transport, heat dissipation, and profiles of heat transfer and thermal reactions. ${ }^{1,} 2$ Furthermore, the precise discrimination of the temperature of a 20 living cell, especially that of a cancer cell, strongly impacts on the consciousness of its pathology and physiology and, in turn, on the optimization of therapeutic processes (e.g. in hyperthermal tumour treatment and photodynamic therapy). ${ }^{3-6}$

Conventional temperature sensors are the so-called contact 25 thermometers, in which the measurement of temperature is achieved by heat flow to an invasive probe. ${ }^{7,8}$ The contact nature of such measurements makes traditional liquid-filled and bimetallic thermometers, thermocouples and thermistors not suitable for temperature measurements of fast-moving objects ${ }^{9}$ or 30 in scales below $10 \mu \mathrm{m}$. Therefore a new generation of nanoscaled thermometers is demanded to accurately measure temperature distributions down to the nanoscale regime, where conventional methods are not able to give satisfactory results (e.g. intracellular temperature fluctuations, ${ }^{4}$ temperature at molecular scale, ${ }^{10}$ or in 35 microcircuits $^{11}$ and microfluids ${ }^{8}$ ).

The first approaches to nanothermometry tried to miniaturize the geometrical size of the conventional thermometers. ${ }^{12}$ For example, filled system thermometers are fabricated from nanotubes based on temperature-dependent thermal expansion of 40 liquids $^{13,14}$ and techniques using an atomic force microscope have succeeded in achieving spatial and temperature resolutions down to $\sim 50 \mathrm{~nm}$ and $\sim 1 \mathrm{mK}$, respectively. ${ }^{2,15}$ Whereas nanotubebased systems require a transmission electron microscope for the read-out and calibration and high vacuum conditions, a marked 45 limitation for practical applications, scanning microscopy systems have a read-out rate limited by material surface conditions and probe motion (typically $30 \mathrm{~ms}$ per pixel ${ }^{15}$ ) making real-time temperature mapping unfeasible.

These constraints lead to the development of non-contact 50 thermometry techniques, such as, IR thermography, Raman spectroscopy, thermoreflectance and luminescence. Amongst non-invasive or semi-invasive spectroscopic methods for determining temperature, the temperature dependence of phosphor luminescence (band shape, peak energy, intensity 55 and/or lifetimes) is an accurate alternative technique, working remotely, through an optical detection system, even in biological fluids, strong electromagnetic fields and fast-moving objects. ${ }^{5,8}$, 16-18 This technique (often referred as thermographic phosphor thermometry) offers high detection sensitivity and spatial 60 resolution in short acquisition times. Thermal probes, such as organic dyes, ${ }^{10,19,20}$ polymers, ${ }^{4,21}$ semiconductor nanocrystals ${ }^{22}$, ${ }^{23}$ and trivalent lanthanides $\left(\mathrm{Ln}^{3+}\right),{ }^{5,15-18,24-34}$ are incorporated into inorganic, organic or organic/inorganic hybrid hosts and most of the luminescent thermometers work exploiting the drop of their ${ }_{65}$ emission intensity with increasing temperature, due to the thermal activation of nonradiative deactivation pathways. A pioneering example is the work of O. Zohar et al. on the thermal imaging of metabolic heat signals generated by ligand-receptor interactions in single cells using the temperature dependent luminescence 70 intensity of $\mathrm{E} \mathrm{Eu}^{3+} \beta$-diketonate chelate. ${ }^{3}$

The present review describes current progress of non-invasive (or semi-invasive) $\mathrm{Ln}^{3+}$-containing luminescent molecular thermometers. Particular emphasis is placed on the few examples that effectively illustrate the temperature sensing/mapping at the 75 sub-micrometer scale: (i) a scanning thermal microscope with $\mathrm{Er}^{3+} / \mathrm{Yb}^{3+}$ co-doped fluoride glass ${ }^{33}$ or $\mathrm{PbF}_{2}$ nanoparticles (NPs) ${ }^{15}$ glued at the tip extremity; (ii) $2 \mathrm{D}$ distribution maps using ${ }^{5} \mathrm{D}_{0}$ rise time temporal response in $\mathrm{Y}_{2} \mathrm{O}_{3}: \mathrm{Eu}^{3+} ;{ }^{29}$ (iii) $\mathrm{Y}_{3} \mathrm{Al}_{5} \mathrm{O}_{12}$ (YAG): $\mathrm{Ce}^{3+} 25$ and $\mathrm{NaYF}_{4}: \mathrm{Er}^{3+} / \mathrm{Yb}^{3+} \mathrm{NPs},{ }^{5}$ (iv) siloxane-based 80 organic-inorganic hybrid NPs incorporating a $\mathrm{Eu}^{3+} \operatorname{tris}(\beta-$ diketonate) complex, ${ }^{17}$ and (v) magnetic hybrid NPs co-doped with $\mathrm{Eu}^{3+}$ and $\mathrm{Tb}^{3+}$ tris( $\beta$-diketonate $)$ chelates. ${ }^{18,35}$

\section{Examples of optical micro and nanothermometers}

Temperature sensing with thermographic phosphors is commonly 85 classified in decay time and intensity ratio algorithms. ${ }^{16}$ Decay time methods exploit the temperature dependence of the lifetime of an emitting level and the intensity ratio algorithms use directly the intensity of one or more transitions to detect temperature. The intensity of each transition is proportional to the total number of 90 atoms (population) in a given excited state at temperature $T$ :

$I \propto g A h v \exp \left(-\frac{E}{k_{B} T}\right)$ 
where $g$ is the degeneracy of the state, $A$ is the spontaneous emission rate, $v$ is the frequency, $h$ and $k_{B}$ are the Planck and the Boltzmann constants, respectively, and $E$ is the energy of the level. Frequently two transitions assigned to the same phosphor 5 are used and the ratio between their fluorescent intensities $\left(I_{l}\right.$ and $I_{2}$ ) taken as a measurement of absolute temperature, since:

$\frac{I_{1}}{I_{2}}=\frac{g_{1} A_{1} h v_{1}}{g_{2} A_{2} h v_{2}} \exp \left(-\frac{\Delta E_{12}}{k_{B} T}\right)=B \times \exp \left(-\frac{\Delta E_{12}}{k_{B} T}\right)$,

10 where $B$ is a constant. The main advantage of using the ratio between the fluorescent intensities of two transitions as a measurement of absolute temperature, a numerical technique known as fluorescence intensity ratio (FIR), is the fact that one single emission spectrum contains all the information needed to 15 compute the absolute temperature. The FIR ratio (sometimes also called two-colour response) is based on the temperature dependent Boltzmann distribution of electrons between adjacent emitting energy levels and manifests as a temperature-dependent intensity ratio between emissions from two distinct spectral lines. 20 The FIR algorithm (as well as the decay time data) makes the temperature sensing independent of the concentration of the sensor and of the drifts of the optoelectronic system (lamp and detectors), thus overcoming the main drawbacks of the intensitybased measurements of only one transition. ${ }^{17,18}$

\section{Scanning thermal microscopes}

The scanning thermal microscope developed by Aigouy and collaborators $^{15,33,36}$ can measure the heating of electrically excited stripes, micro and nanowires (i.e. $1 \mu \mathrm{m}$-wide, $40 \mu \mathrm{m}$-long 30 and $40 \mathrm{~nm}$ thick nickel stripe) by gluing $\mathrm{Er}^{3+} / \mathrm{Yb}^{3+}$ co-doped fluoride amorphous glass particles or $\mathrm{PbF}_{2}: \mathrm{Er}^{3+} / \mathrm{Yb}^{3+}$ nanocrystals at the extremity of an atomic force microscope scanning tip (Fig. 1). The $\mathrm{Yb}^{3+}$ ions were excited by a $975 \mathrm{~nm}$ near infrared (NIR) laser, through an NIR-to-visible up-conversion (UC) process in 35 which two low energy photons are "added up" to give one high energy photon. ${ }^{37}$ This NIR excitation is very efficient because, at this wavelength, the $\mathrm{Yb}^{3+}$ ions have a large absorption cross section. Once excited, they transfer their energy to adjacent $\mathrm{Er}^{3+}$ ions that will glow in the green $\left({ }^{2} \mathrm{H}_{11 / 2} \rightarrow{ }^{4} \mathrm{I}_{15 / 2}\right.$ and ${ }^{4} \mathrm{~S}_{3 / 2} \rightarrow{ }^{4} \mathrm{I}_{15 / 2}, c a$. 40525 and $550 \mathrm{~nm}$, respectively) and red $\left({ }^{4} \mathrm{~F}_{9 / 2} \rightarrow{ }^{4} \mathrm{I}_{15 / 2}, c a .660 \mathrm{~nm}\right)$ spectral regions. These $\mathrm{Er}^{3+}$ transitions (that are insensitive to photodegradation) are temperature dependent and their intensities were used to determine the temperature using the FIR algorithm.

By adjusting the electrical current flowing through the 45 structure, the resulting temperature variations modulate the $\mathrm{Er}^{3+}$ particle fluorescence giving rise to the thermal contrast. Although the fluorescence is affected both by the near-field optical distribution and by temperature variations, this thermal contribution can be treated separately by comparing the obtained 50 images with reference ones recorded when the current in the device is zero. The determination of the temperature is, therefore, performed by analyzing the thermal quenching of the $\mathrm{Er}^{3+}$ fluorescence. The results obtained are in good agreement with numerical simulations of the heat dissipation of the micro and 55 nanowires. Since the fluorescent probe is not in direct contact with the micro or nanowires, the temperature determined by this method is actually the average temperature of the fluorescent probe that is controlled by the temperature of the interaction volume between the NP and the surface of the wires.

60 For the scanning thermal microscope using $\mathrm{PbF}_{2}: \mathrm{Er}^{3+} / \mathrm{Yb}^{3+}$ NPs as fluorescence probes ${ }^{15}$ the relative sensitivity is $1.1 \% / \mathrm{K}$ at $\sim 310 \mathrm{~K}$ (Table 1). This thermometer presents a spatial resolution (defined as the minimum distance to move in order to get a temperature change greater than the sensitivity of the 65 thermometer $)$ in the range of the fluorescent particle size $(<500$ $\mathrm{nm})$. For the scanning thermal microscope with the $\mathrm{Er}^{3+} / \mathrm{Yb}^{3+}$ codoped fluoride amorphous glass particles, ${ }^{33}$ the relative sensitivity changes between 0.37 and $0.59 \% / \mathrm{K}$, in the temperature range 297-390 K (Table 1). While in this latter scanning thermal 70 microscope the quenching of luminescence with temperature is calculated directly from the temperature dependence of the intensity of the two ${ }^{2} \mathrm{H}_{11 / 2} \rightarrow{ }^{4} \mathrm{I}_{15 / 2}$ and ${ }^{4} \mathrm{~S}_{3 / 2} \rightarrow{ }^{4} \mathrm{I}_{15 / 2}$ transitions, ${ }^{33}$ in the former example, ${ }^{15}$ the quenching value is extracted from the normalized thermally modulated fluorescence images.

75 Tikhomirov et al. used an analogous UC mechanism to fabricate an optical nanoheater of fluoride $\mathrm{Yb}^{3+} / \mathrm{Er}^{3+}$ co-doped NPs embedded into different host matrices. ${ }^{32}$ The NPs were excited by NIR radiation (laser diode at $975 \mathrm{~nm}$ ) that is dissipated via phonon relaxation (up conversion process), resulting in a ${ }_{80}$ temperature increase, measured by FIR of transitions of $\mathrm{Er}^{3+}$ $\left({ }^{2} \mathrm{H}_{11 / 2} \rightarrow{ }^{4} \mathrm{I}_{15 / 2}\right.$ and $\left.{ }^{4} \mathrm{~S}_{3 / 2} \rightarrow{ }^{4} \mathrm{I}_{15 / 2}\right)$, until $800^{\circ} \mathrm{C}$. The authors have suggested the use of the nanoheater for local hypothermal treatment of cells with simultaneous monitoring of temperature.

\section{${ }_{85}$ Upconverting nanoparticles}

The rise time response of the ${ }^{5} \mathrm{D}_{0}$ excited state in $\mathrm{Y}_{2} \mathrm{O}_{3}: \mathrm{Eu}^{3+}$ (3-4\% mole concentration) phosphor was calibrated against a temperature range of $200-700{ }^{\circ} \mathrm{C}$ demonstrating 2D rise time thermal imaging over an area of $\sim 500 \mathrm{~mm}^{2}$ (diameter of the laser 90 beam on the target). ${ }^{29}$ The uncertainty in the temperature measurements is relatively high, $\sim 20 \%$ at $673 \mathrm{~K}$, although a decrease of the $\mathrm{Eu}^{3+}$ activator concentration could significantly lengthen the rise time and then the accuracy of the thermometer.

The sensitivity and the spatial resolution were not reported and 95 the temperature determination algorithm is rather complicated, relatively to those of decay time and intensity-based measurements, requiring more sophisticated equipment and postprocessing computational analysis. ${ }^{29}$

Other example that illustrates the sensing/mapping of 100 temperature at the sub-micrometer scale was reported by Allison et $a .^{25}$ The laser-induced fluorescence lifetime of YAG:Ce NPs (average size $\sim 30 \mathrm{~nm}$ ) was measured as a function of temperature from 280 to $350 \mathrm{~K}$. The decay lifetimes for the NPs varied from 18 to $27 \mathrm{~ns}(\sim 33 \%$ relative to the longest lifetime measured) and 105 this significant variation, coupled with the high signal strength that was observed, suggested the utilisation of YAG:Ce NPs as useful thermographic nanophosphors. From an instrumentation perspective, the short decay times of Ce-doped nanophosphors makes the fluorescence signal easier to acquire and analyse, 110 relatively to phosphors with millisecond decay times (when roughly the same number of photons are emitted and detected in both cases), thus providing a noteworthy advantage. The reason 
for this improvement is that the peak values of the signals are greater and the rise times are faster, thus reducing triggering errors in the detection electronics. Another attractive characteristic of Ce-doped nanophosphors is that they can 5 typically be excited by inexpensive light emitting diodes, thus not only reducing the cost of the phosphor thermography system but also alleviating any concerns about heating of the phosphor substrate by the laser (with the resulting introduction of a systematic uncertainty in the temperature measurements). ${ }^{25}$

10 Vetrone et al. devised a nanothermometer based on the temperature-sensitive upconversion (UC) green emission of $\mathrm{NaYF}_{4}: \mathrm{Er}, \mathrm{Yb}$ NPs (mean particle size of $\sim 18 \mathrm{~nm}$ ). ${ }^{5}$ The upconverting $\mathrm{Ln}^{3+}$-doped NPs can be stimulated using low power and inexpensive NIR lasers and are excited with wavelengths in

15 the optical penetration window of cells and tissues (650-1300 $\mathrm{nm}$ ), an important requirement in biological environments who suffer less damage under excitation in such range. Moreover, besides less scattered by the specimen, NIR excitation light will not induce background autofluorescence, leading to increased 20 signal-to-noise ratio of the detected signal.

Thermal profiles created when heating a colloidal solution of the $\mathrm{NaYF}_{4}: \mathrm{Er}, \mathrm{Yb}$ NPs in water using a pump-probe experiment were obtained using the temperature dependence of the intensity ratio between the $\mathrm{Er}^{3+}{ }^{2} \mathrm{H}_{11 / 2} \rightarrow{ }^{4} \mathrm{I}_{15 / 2}$ and ${ }^{4} \mathrm{~S}_{3 / 2} \rightarrow{ }^{4} \mathrm{I}_{15 / 2}$ transitions. ${ }^{5}$ 25 The internalization of the NPs by human cervical carcinoma (HeLa) cells permits to exploit the thermal sensitivity of the two $\mathrm{Er}^{3+}$ bands to create a nanothermometer capable of measuring the internal temperature of a living cancer cell, from $298 \mathrm{~K}$ to its thermally induced death at $318 \mathrm{~K}$. The cells were placed in a 30 confocal fluorescence microscope and excited at $920 \mathrm{~nm}$ so that the upconverted $\mathrm{Er}^{3+}$ fluorescence of the NPs permitted the measurement of the inner HeLa cell temperature (Fig. 2). The $\mathrm{NaYF}_{4}: \mathrm{Er}^{3+} / \mathrm{Yb}^{3+} \mathrm{NPs}$ are one of the few cases reported so far of cellular thermometers. ${ }^{3,4}$ Despite the absence of a quantitative 35 estimation of the thermometer sensitivity, the NPs are able to investigate (without using an exterior reference) the cellular changes occurring between 298 and $318 \mathrm{~K}$, as a result of the external heating.

\section{${ }_{40}$ Organic-Inorganic hybrids}

The last two examples illustrating the sensing/mapping of temperature at the nanoscale report the incorporation of $\mathrm{Ln}^{3+}$ based $\beta$-diketonate complexes into organic-inorganic hybrid materials. ${ }^{17,18}$ Luminescent molecular thermometers based on ${ }_{45} \mathrm{Eu}^{3+}$ tris( $\beta$-diketonate) complexes were proposed in the past decades involving isolated complexes ${ }^{24}, 38$ or complexes embedded into polymer thin films ${ }^{28,39}$ and organic-inorganic hybrid materials ${ }^{17,} 18$ (maximum sensitivities and temperature ranges listed in Table 1). The complexes act as temperature 50 sensors through a thermally driven energy back transfer mechanism between the ${ }^{5} \mathrm{D}_{0} \mathrm{Eu}^{3+}$ emitting level and the lowest ligand triplet excited state. Then, its thermal sensitivity can be tuned by changing the ligands and/or the metal ion concentration. ${ }^{24,} 38$ A serious drawback of $\mathrm{Ln}^{3+} \beta$-diketonate 55 complexes relates to their photodecomposition under UV irradiation (in certain cases, in only a few hours), ${ }^{40}$ which decreases luminescence intensity and, thus, makes molecular thermometers based on these complexes not suitable for longterm monitoring. However, the incorporation of the complexes 60 into organic-inorganic hybrid hosts considerably improves the UV photostability, ${ }^{40}$ overcoming, therefore, the main drawback of the use of such complexes as long-term monitoring luminescent thermometers. Furthermore, the thermal stability and the mechanical properties of the resulting $\mathrm{Ln}^{3+}$-containing organic65 inorganic hybrids are considerably ameliorated, relatively to those of the parent complexes. This is an important advantage concerning the technological applicability of these materials, namely in lighting, sensing, and displays. Finally, the combination of the intrinsic characteristics of sol-gel derived 70 hosts and the luminescence features of $\mathrm{Ln}^{3+}$ ions in multifunctional organic-inorganic hybrids offer excellent perspectives for designing new luminescent materials with enhanced desired characteristics, thus opening exciting new directions in materials science and related technologies, with 75 noteworthy results in the ecofriendly integration, miniaturization, and multifunctionalization of devices. ${ }^{40,41}$

Peng et al. ${ }^{17}$ reported the incorporation of the Eu-DT complex (DT stands for tris(dinaphthoylmethane)-bis-(trioctylphosphine oxide) into siloxane hybrid NPs (formed by a BTD-PMMA 80 hybrid matrix with a silica outer layer, BTD and PMMA represents, respectively, 2-bis(trimethoxysilyl)decane and poly(methylmethacrylate)). The NPs, with size ranging from 20 to $30 \mathrm{~nm}$, display strong temperature dependence, in both luminescence intensity and ${ }^{5} \mathrm{D}_{0}$ lifetime, over the physiological ${ }_{85}$ range $(298-318 \mathrm{~K})$. In fact, the intensity of the ${ }^{5} \mathrm{D}_{0} \rightarrow{ }^{7} \mathrm{~F}_{2}$ transition decreases by $-3.07 \%$ per degree, on increasing temperature from 298 to $318 \mathrm{~K}$ (Table 1 ). The ${ }^{5} \mathrm{D}_{0}$ lifetime dropped rapidly with the increase of temperature in that range displaying a temperature sensitivity of $2.2 \%$ per degree (Table 1 ). 90 As mentioned above (and explicitly recognized by the authors in ref. ${ }^{17}$ ), intensity measurements based on one transition depend critically on the variations of the sensor concentration and of the optoelectronic drifts of the excitation source (normalization is necessary) and detectors, thus rendering inappropriate for precise 95 temperature sensing. Lifetime data and FIR measurements, in contrast, are not compromised by those drawbacks and are therefore much more reliable. Nevertheless, we should stress that the decay time algorithm is unsuitable for real-time temperature sensing and mapping, requiring more sophisticated equipment, 100 when compared to the faster, easier and cheaper FIR method. Precise and real-time in vivo monitoring of temperature is of paramount importance, for instance, in biomedicine, namely in cancer diagnosis and during hypothermia therapy or surgery where temperature fluctuations of a few degrees can be telling. ${ }^{10}$ 105 Moreover, for complicated distributions of emitting centres and/or strong interactions between them (for instance energy transfer) the procedure may be laborious and time-consuming, as lifetime determination involves a fit procedure to the decay curves. Therefore, it is unquestionable that an optimal 110 luminescent nanothermometer should produce a ratiometric intensity response to temperature changes (the FIR algorithm).

Examples of self-referencing luminescent molecular thermometers include the temperature-dependent of perylene monomer-perylene/N-allyl-N-methylaniline exciplex 115 interconversion ${ }^{19}$ and monomer-excimer interconversion of 1,3- 
bis(1-pyrenyl)propane methylpyrrolidinium

dissolved in the 1-butyl-1bis(trifluoromethylsulfonyl)imide, $\left.\left[\mathrm{C}_{4} \mathrm{mpy}\right]\left[\mathrm{Tf}_{2} \mathrm{~N}\right]\right)$, ionic liquid, ${ }^{10}$ for dye-doped materials, and the ${ }^{2} \mathrm{H}_{11 / 2} \rightarrow{ }^{4} \mathrm{I}_{15 / 2} /{ }^{4} \mathrm{~S}_{3 / 2} \rightarrow{ }^{4} \mathrm{I}_{15 / 2}$ intensity ratio, in $\mathrm{Er}^{3+} / \mathrm{Yb}^{3+}$-based up5 converting NPs, ${ }^{5,15,30-32,42,43}$ and the ${ }^{5} \mathrm{D}_{0} \rightarrow{ }^{7} \mathrm{~F}_{2} /{ }^{5} \mathrm{D}_{4} \rightarrow{ }^{7} \mathrm{~F}_{5}$ intensity ratio in $\mathrm{Eu}^{3+} / \mathrm{Tb}^{3+}$-doped hybrid magnetic nanoclusters, ${ }^{18}$ for $\mathrm{Ln}^{3+}$-based materials.

This latter example consists of a luminescent molecular thermometer based on hybrid magnetic nanoclusters (size ranging 10 from 100 to $400 \mathrm{~nm}$ ) formed by a $\gamma-\mathrm{Fe}_{2} \mathrm{O}_{3}$ maghemite core (hydrodynamic size of $21.0 \pm 4.0 \mathrm{~nm}$ ) coated with a tetraethyl orthosilicate/aminopropyltriethoxysilane

(TEOS/APTES) organosilica shell and co-doped with $\left[\mathrm{Eu}(\mathrm{btfa})_{3}(\mathrm{MeOH})(\mathrm{bpeta})\right]$ and $\left[\mathrm{Tb}(\mathrm{btfa})_{3}(\mathrm{MeOH})(\right.$ bpeta) $] \beta$-diketonate chelates [where btfa', 15 bpeta and $\mathrm{MeOH}$ represent, respectively, 4,4,4-trifluoro-1phenyl-1,3-butanedionate, 1,2-bis(4-pyridyl)ethane and methanol], Fig. 3. ${ }^{18,} 35$ The $\mathrm{Eu}^{3+} / \mathrm{Tb}^{3+}$ co-doped NPs were prepared using Eu:Tb ratios of 2:1, 1:1, 1:2, 1:3 and 1:10 followed the procedure described elsewhere, supporting 20 information of ref. ${ }^{17}$.

The $\mathrm{Eu}^{3+} / \mathrm{Tb}^{3+}$ luminescent nanothermometer is selfreferencing, allowing absolute measurements in the $10-350 \mathrm{~K}$ temperature range with a temperature uncertainty of 0.5 degree. Its emission colour is fine-tuned as a function of temperature 25 (Fig. 4) and the temperature sensitivity is up to $4.9 \% \cdot \mathrm{K}^{-1}$, the highest value reported so far for $\mathrm{Ln}^{3+}$-based thermometers. Moreover, it exhibits high photostability in long-term use.

Highly luminescent water suspensions of the hybrid $\mathrm{Eu}^{3+} / \mathrm{Tb}^{3+}$ NPs at room temperature (Fig. 5) indicate that the quenching of 30 the $\mathrm{Ln}^{3+}$ emission by nonradiative energy transfer from the intra$4 f$ excited states to the high-energy $\mathrm{OH}$-vibrations $\left(\sim 3700 \mathrm{~cm}^{-1}\right)$ is not significant. The $\mathrm{Eu}^{3+}$ and $\mathrm{Tb}^{3+}$ first coordination ligands in these NPs efficiently shield the metal centres from the $\mathrm{OH}$ oscillations permitting, therefore, that the nanothermometer 35 operates in aqueous suspensions, a critical advantage for biomedical applications.

The variation of the $\mathrm{Eu}^{3+} / \mathrm{Tb}^{3+}$ ratio affords tunability to the temperature working range. Alternatively, tunability is also accomplished by changing the host matrix, thus modifying the 40 interaction between the $\mathrm{Ln}^{3+}$ and the host matrix energy levels. ${ }^{18}$ This is the case of $\mathrm{Eu}^{3+} / \mathrm{Tb}^{3+}$ co-doped di-ureasils ${ }^{40}$ that permitted absolute temperature measurements at physiological temperatures. Moreover, the di-ureasil hybrid matrix enables processing the thermometer material as thin films for 45 sensing/mapping large areas with a spatial resolution limited by the size of the optical detectors $(\sim 1-10 \mu \mathrm{m}$ for commercial optical fibres and CCD cameras). Finally, the combination of the molecular thermometer with a nanometric magnetic/luminescent host matrix provides multifunctionality at the nanoscale to the 50 device.

When comparing this thermometer with the single $\mathrm{Ln}^{3+}$-based ones, proposed previously, it is clear that it represents a step forward in thermometry at the nanoscale. The synergy resulting from combining temperature sensing/mapping and 55 superparamagnetism opens the way for new exciting applications, especially in the biomedical field. In particular, such association will provide a unique instrument to map, in a non-invasive way, temperature distributions in biological tissues (e.g., in tumours) during heat release, due to the application of an ac field to 60 magnetic NPs (magnetic hyperthermia), this being, undoubtedly, a powerful tool for the study of biochemical micro-processes occurring within a cell.

\section{Conclusions}

This review summarized the recent progress on non-invasive ${ }_{65} \mathrm{Ln}^{3+}$-containing luminescent molecular thermometers, with particular emphasis on the handful examples reported so far that effectively illustrate the temperature sensing/mapping at the submicrometer scale: scanning thermal microscopes that uses emitter NPs glued at the end of the scanning tip as temperature probes; $70 \mathrm{NPs}\left(\mathrm{Y}_{2} \mathrm{O}_{3}: \mathrm{Eu}^{3+}\right.$, YAG: $\mathrm{Ce}^{3+}$ and $\left.\mathrm{NaYF}_{4}: \mathrm{Er}^{3+} / \mathrm{Yb}^{3+}\right)$, and $\mathrm{Eu}^{3+}$ - or $\mathrm{Eu}^{3+} / \mathrm{Tb}^{3+}$-containing siloxane-based organic-inorganic hybrid NPs. The main challenges that are currently facing scientists in the field are: (i) synthesis of biocompatible and highly efficient luminescent up-converting NPs acting as thermometers capable 75 of operate in the physiological temperature range with submicron spatial resolution,; (ii) design of different host hybrid matrices and/or lanthanide ligands to make absolute measurements at the physiological temperature range feasible with hybrid NPs; (iii) internalization of the NPs (both the up80 converting and the hybrid ones) into cellular medium; (iv) quantitative characterization of the NP-to-surface heat transfer mechanisms, particularly for scanning thermal microscopes; and (v) combination and integration of different functionalities into more complex architectures, e.g. magnetic hyperthermia and the 85 corresponding temperature monitoring. This sort of "lab-on-aparticle" technology is clearly one of the next evolutionary steps in this research area with dramatic impact on information and communication technologies, global health and biomedicine.

\section{Acknowledgements}

${ }_{90}$ The authors are grateful to Fundação para a Ciência e a Tecnologia (FCT, Portugal), COMPETE and FEDER programs (PTDC/CTM/101324/2008) and Integrated SpanishPortuguese Action PT2009-0131 for financial support. The work in Zaragoza has been supported by the grants 95 MAT2007-61621 and CONSOLIDER CSD2007-00010 from the Ministry of Education. CDSB (SFRH/BD/38472/2007) and PPL (SFRH/BPD/34365/2006) thank FCT for grants. NJOS acknowledges FCT for Ciência 2008 program.

\section{Notes and references}

$100{ }^{a}$ Department of Physics, CICECO, University of Aveiro, Campus Universitário de Santiago, 3810-193 Aveiro, Portugal. Fax: 351234 378197; Tel: 351234 370946; E-mail: lcarlos@ua.pt

${ }^{b}$ Departamento de Fisica de la Materia Condensada, Facultad de Ciencias and Instituto de Ciencia de Materiales de Aragón, CSIC105 Universidad de Zaragoza, 50009 Zaragoza, Spain.

1. J. Lee and N. A. Kotov, Nano Today, 2007, 2, 48-51.

2. S. Sadat, A. Tan, Y. J. Chua and P. Reddy, Nano Lett., 2010, 10, 2613-2617.

110 3. O. Zohar, M. Ikeda, H. Shinagawa, H. Inoue, H. Nakamura, D. Elbaum, D. L. Alkon and T. Yoshioka, Biophys. J., 1998, 74, 8289. 
4. C. Gota, K. Okabe, T. Funatsu, Y. Harada and S. Uchiyama, J. Am. Chem. Soc., 2009, 131, 2766-2767.

5. F. Vetrone, R. Naccache, A. Zamarron, A. J. de la Fuente, F. Sanz-Rodriguez, L. M. Maestro, E. M. Rodriguez, D. Jaque, J. G.

5 Sole and J. A. Capobianco, Acs Nano, 2010, 4, 3254-3258.

6. H. Huang, S. Delikanli, H. Zeng, D. M. Ferkey and A. Pralle, Nat. Nanotechnol., 2010, 5, 602-606.

7. P. R. N. Childs, J. R. Greenwood and C. A. Long, Rev. Sci. Instrum., 2000, 71, 2959-2978.

10 8. T. Barilero, T. Le Saux, C. Gosse and L. Jullien, Anal. Chem., 2009, 81, 7988-8000.

9. O. S. Wolfbeis, Adv. Mater., 2008, 20, 3759-3763.

10. G. A. Baker, S. N. Baker and T. M. McCleskey, Chem. Commun., 2003, 2932-2933.

15 11. G. Tessier, M. Bardoux, C. Boue and D. Fournier, Appl. Phys. Lett., 2007, 90, 171112

12. Y. C. Lan, H. Wang, X. Y. Chen, D. Z. Wang, G. Chen and Z. F. Ren, Adv. Mater., 2009, 21, 4839-4844.

13. Y. H. Gao and Y. Bando, Nature, 2002, 415, 599-599.

20 14. C. Y. Wang and L. J. Chen, Eur. J. Inorg. Chem. , 2010, 42984303.

15. L. Aigouy, E. Saidi, L. Lalouat, J. Labeguerie-Egea, M. Mortier, P. Low and C. Bergaud, J. Appl. Phys., 2009, 106, 074301.

16. A. L. Heyes, J. Lumin., 2009, 129, 2004-2009.

25 17. H. S. Peng, M. I. J. Stich, J. B. Yu, L. N. Sun, L. H. Fischer and O. S. Wolfbeis, Adv. Mater., 2010, 22, 716-719.

18. C. D. S. Brites, P. P. Lima, N. J. O. Silva, A. Millán, V. S. Amaral, F. Palacio and L. D. Carlos, Adv. Mater., 2010, 22, 4499-4504.

30 19. N. Chandrasekharan and L. A. Kelly, J. Am. Chem. Soc., 2001, 123, 9898-9899.

20. P. Löw, B. Kim, N. Takama and C. Bergaud, Small, 2008, 4, 908.

21. E. M. Graham, K. Iwai, S. Uchiyama, A. P. de Silva, S. W. Magennis and A. C. Jones, Lab Chip, 2010, 10, 1267-1273.

35 22. G. Walker, V. Sundar, C. Rudzinski, A. Wun, M. Bawendi and D. Nocera, Appl. Phys. Lett., 2003, 83, 3555.

23. V. A. Vlaskin, N. Janssen, J. van Rijssel, R. Beaulac and D. R. Gamelin, Nano Lett., 2010, 10, 3670-3674.

24. G. E. Khalil, K. Lau, G. D. Phelan, B. Carlson, M. Gouterman, J.

40 B. Callis and L. R. Dalton, Rev. Sci. Instrum., 2004, 75, 192-206.

25. S. Allison, G. Gillies, A. Rondinone and M. Cates, Nanotechnology, 2003, 14, 859-863.

26. A. L. Heyes, S. Seefeldt and J. P. Feist, Opt. Laser Technol., 2006, 38, 257-265.

45 27. H. Aizawa, T. Katsumata, S. Komuro, T. Morikawa, H. Ishizawa and E. Toba, Sensor Actuat. A-Phys., 2006, 126, 78-82.

28. S. Katagiri, K. Manseki, Y. Tsukahara, K. Mitsuo and Y. Wada, J. Alloy. Compd., 2008, 453, L1-L3.

29. A. H. Khalid and K. Kontis, Meas. Sci. Technol., 2009, 20, $50 \quad 025305$.

30. S. K. Singh, K. Kumar and S. B. Rai, Sensor Actuat. A-Phys., 2009, 149, 16-20.

31. S. Singh, K. Kumar and S. Rai, Appl. Phys. B: Lasers Opt., 2009, 94, 165-173.

55 32. V. Tikhomirov, K. Driesen, V. Rodriguez, P. Gredin, M. Mortier and V. Moshchalkov, Opt. Express, 2009, 17, 11794-11798.

33. E. Saïdi, B. Samson, L. Aigouy, S. Volz, P. Löw, C. Bergaud and M. Mortier, Nanotechnology, 2009, 20, 115703.

34. J. B. Yu, L. N. Sun, H. S. Peng and M. I. J. Stich, J. Mater. 60 Chem., 2010, 20, 6975-6981.

35. F. Palacio, A. Millán, N. J. Silva, L. D. Carlos, V. Amaral, P. P. Lima and C. D. S. Brites, Spain Patent, 2009, P200930367.

36. L. Aigouy, G. Tessier, M. Mortier and B. Charlot, Appl. Phys. Lett., 2005, 87, 184105

65 37. F. Wang and X. G. Liu, Chem. Soc. Rev., 2009, 38, 976-989.

38. S. Uchiyama, A. P. de Silva and K. Iwai, J. Chem. Educ., 2006, 83, 720-727.

39. M. Mitsuishi, S. Kikuchi, T. Miyashita and Y. Amao, J. Mater. Chem., 2003, 13, 2875-2879.

70 40. L. D. Carlos, R. A. S. Ferreira, V. de Zea Bermudez and S. J. L. Ribeiro, Adv. Mater., 2009, 21, 509-534.

41. C. Sanchez, B. Julian, P. Belleville and M. Popall, J. Mater. Chem., 2005, 15, 3559-3592.

42. X. Wang, X. G. Kong, Y. Yu, Y. J. Sun and H. Zhang, J. Phys. 75 Chem. C, 2007, 111, 15119-15124.

43. M. A. R. C. Alencar, G. S. Maciel, C. B. de Araujo and A. Patra, Appl Phys Lett, 2004, 84, 4753-4755.

44. B. Zelelow, G. E. Khalil, G. Phelan, B. Carlson, M. Gouterman, J. B. Callis and L. R. Dalton, Sensor Actuat. B-Chem., 2003, 96, $80 \quad 304-314$. 
Fig. 1 Exemple of the eperimental setup used in scanning thermal microscopes. The device is powered by a square electrical current (brown curve) that induces a periodic Joule heating (red curve) and a fluorescence quenching of the 5 particle (black curve). The inset is a scanning electron microscope image of the nanocrystal glued at the end of the tip. (Reproduced with permission from ref. ${ }^{15}$, copyright 2009 AIP).

${ }_{10}$ Fig. 2 (Top) Optical transmission images of an individual HeLa cell at three inner temperatures. Cell death is observed at $45{ }^{\circ} \mathrm{C}$. (Bottom) Temperature of the HeLa cell determined by $\mathrm{Er}^{3+}$ luminescence in the $\mathrm{NaYF}_{4}: \mathrm{Er}, \mathrm{Yb} \mathrm{NPs}$ as a function of the applied voltage. (Reproduced with permission from ref. $15^{5}$, copyright 2010 ACS).

Fig. 3 (a) Optical microscope image at $300 \mathrm{~K}$ (UV excitation at $365 \mathrm{~nm}$ ) of NP3.1-2 NPs. The images were recorded in a Carl Zeiss Axiovert $200 \mathrm{M}$ fluorescence microscope equipped 20 with Apotome at 125 and $300 \mathrm{~K}$. The red colour corresponds to real colour. (b) SEM image with a general view of the NPs. (c) Amplification TEM image of the NPs showing aggregation occurred probably during the evaporation of the dispersion on the grid.

25

Fig. 4 (Top) Optical microscope images at 125 and $300 \mathrm{~K}$ (UV excitation at $365 \mathrm{~nm}$ ) of NP3.1-2 NPs. The colours correspond to real colours. (Bottom) Emission spectra (excited at $365 \mathrm{~nm}$ ) of the NPs recorded at 125 and $300 \mathrm{~K}$.

30

Fig. 5 Photograph of an water suspension (1 $\mathrm{mL})$ of the NP3.1-2 NPs under (a) day light illumination and (b) UV excitation $(365 \mathrm{~nm})$ throughout an optical fibre bundle. 
Table 1 - Sensitivity and temperature operation range of $\mathrm{Ln}^{3+}$-based luminescent molecular thermometers.

\begin{tabular}{|c|c|c|c|c|c|}
\hline Phosphor & \begin{tabular}{c}
\multicolumn{1}{c}{ Maximum } \\
Sensitivity \\
(\% Intensity / K)
\end{tabular} & $\begin{array}{l}\text { Temperature } \\
\text { range }(\mathbf{K})\end{array}$ & $\begin{array}{l}\text { Temperature of maximum } \\
\text { sensitivity }(\mathbf{K})\end{array}$ & $\begin{array}{l}\text { Temperature dependence } \\
\text { algorithm }\end{array}$ & Observations \\
\hline $\mathrm{BaTiO}_{3}: \mathrm{Er} \mathrm{NPs}^{43}$ & $0.5^{\text {(a) }}$ & $333-466$ & 333 & $\begin{array}{l}{ }^{2} \mathrm{H}_{11 / 2} \rightarrow{ }^{4} \mathrm{I}_{15 / 2} /{ }^{4} \mathrm{~S}_{3 / 2} \rightarrow{ }^{4} \mathrm{I}_{15 / 2} \\
\text { intensity ratio }\end{array}$ & $\begin{array}{l}\text { Effect of the size of the NP on sensitivity } \\
\text { discussed }\end{array}$ \\
\hline $\mathrm{ZnO}: \mathrm{Er} \mathrm{NPs}^{42}$ & $0.6^{(\mathrm{a})}$ & $283-475$ & $\sim 443$ & $\begin{array}{c}{ }^{2} \mathrm{H}_{11 / 2} \rightarrow{ }^{4} \mathrm{I}_{15 / 2} /{ }^{4} \mathrm{~S}_{3 / 2} \rightarrow{ }^{4} \mathrm{I}_{15 / 2} \\
\text { intensity ratio }\end{array}$ & 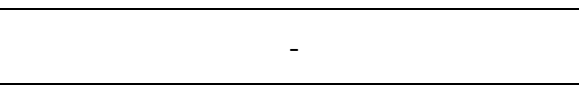 \\
\hline $\mathrm{Gd}_{2} \mathrm{O}_{3}: \mathrm{Er} / \mathrm{Yb}^{30,31}$ & $0.4^{(\mathrm{b})}$ & $300-900$ & $\sim 300$ & $\begin{array}{l}{ }^{2} \mathrm{H}_{11 / 2} \rightarrow{ }^{4} \mathrm{I}_{15 / 2} /{ }^{4} \mathrm{~S}_{3 / 2} \rightarrow{ }^{4} \mathrm{I}_{15 / 2} \\
\text { intensity ratio }\end{array}$ & ( \\
\hline $\begin{array}{l}\mathrm{Er} / \mathrm{Yb} \text { co-doped } \mathrm{PbF}_{2} \\
\mathrm{NPs}^{15}\end{array}$ & $1.1^{\text {(a) }}$ & $290-325$ & $\sim 310$ & $\begin{array}{l}{ }^{2} \mathrm{H}_{11 / 2} \rightarrow{ }^{4} \mathrm{I}_{15 / 2} /{ }^{4} \mathrm{~S}_{3 / 2} \rightarrow{ }^{4} \mathrm{I}_{15 / 2} \\
\text { intensity ratio }\end{array}$ & $\begin{array}{c}\text { Accuracy of } 0.8 \mathrm{~K} \text {. For the } \mathrm{Er}^{3+} / \mathrm{Yb}^{3+} \mathrm{co}- \\
\text { doped fluoride amorphous glass NPs } \\
\text { relative sensitivity changes between } 0.37 \text { and } \\
0.59 \% / \mathrm{K}\end{array}$ \\
\hline Eu $\beta$-diketonate complex ${ }^{28}$ & $\sim 2.0^{\text {(a) }}$ & $280-330$ & $\sim 283$ & ${ }^{5} \mathrm{D}_{0} \rightarrow{ }^{7} \mathrm{~F}_{2}$ intensity & - \\
\hline Eu/Tb hybrid NPs ${ }^{18}$ & $4.7^{\text {(a) }}$ & $10-350$ & 134 & $\begin{array}{c}{ }^{5} \mathrm{D}_{0} \rightarrow{ }^{7} \mathrm{~F}_{2} /{ }^{5} \mathrm{D}_{4} \rightarrow{ }^{7} \mathrm{~F}_{5} \text { intensity } \\
\text { ratio }\end{array}$ & $\begin{array}{l}\text { The Eu/Tb-containing di-ureasil film presents } \\
\text { a sensitivity of } 0.6 \% / \mathrm{K} \text { from } 298 \text { to } 318 \mathrm{~K}\end{array}$ \\
\hline Eu hybrid NPs ${ }^{17}$ & $3.1^{(\mathrm{a})}$ & 283-323 & $\sim 283$ & $\begin{array}{c}{ }^{5} \mathrm{D}_{0} \rightarrow{ }^{7} \mathrm{~F}_{2} \text { intensity and }{ }^{5} \mathrm{D}_{0} \\
\text { lifetime }\end{array}$ & $\begin{array}{l}\text { Temperature resolution of } \sim 0.3 \mathrm{~K} \text {, assuming a } \\
\text { precision of } 1 \% \text { in emission intensity }\end{array}$ \\
\hline Eu and $\mathrm{Tb}$ complexes ${ }^{34}$ & $1.2^{(\mathrm{a})}$ & 295-335 & - & $\begin{array}{c}{ }^{5} \mathrm{D}_{4} \rightarrow{ }^{7} \mathrm{~F}_{5}\left(\mathrm{~Tb}^{3+}\right) \text { and } \\
{ }^{5} \mathrm{D}_{0} \rightarrow{ }^{7} \mathrm{~F}_{2}\left(\mathrm{Eu}^{3+}\right) \text { intensities; } \\
{ }^{5} \mathrm{D}_{4} \text { and }{ }^{5} \mathrm{D}_{0} \text { lifetimes }\end{array}$ & $\begin{array}{l}\text { The maximum sensitivity is reported for the } \\
\text { Tb complex in a solution of dimethyl } \\
\text { sulfoxide. Maximum }{ }^{5} \mathrm{D}_{4} \text { lifetime sensitivity } \\
\text { of } 13.8 \mu \mathrm{s} / \text { degree ( } 3 \mathrm{wt} \% \text { in PMMA); } \\
\text { Pressure sensor for the Eu complex }\end{array}$ \\
\hline $\begin{array}{l}\text { Eu } \beta \text {-diketonate and } \mathrm{Pt} \\
\text { porphyrin type } \\
\text { complexes }^{24,44}\end{array}$ & $0.9-4.4^{(\mathrm{b})}$ & $278-323$ & $\sim 278$ & $\begin{array}{l}{ }^{5} \mathrm{D}_{0} \rightarrow{ }^{7} \mathrm{~F}_{2} \text { intensity and }{ }^{5} \mathrm{D}_{0} \\
\text { lifetime }\end{array}$ & $\begin{array}{c}\text { The two luminophors were jointly } \\
\text { incorporated into a polymer single paint } \\
\text { allowing temperature and pressure } \\
\text { measurements }\end{array}$ \\
\hline
\end{tabular}


Figure 1

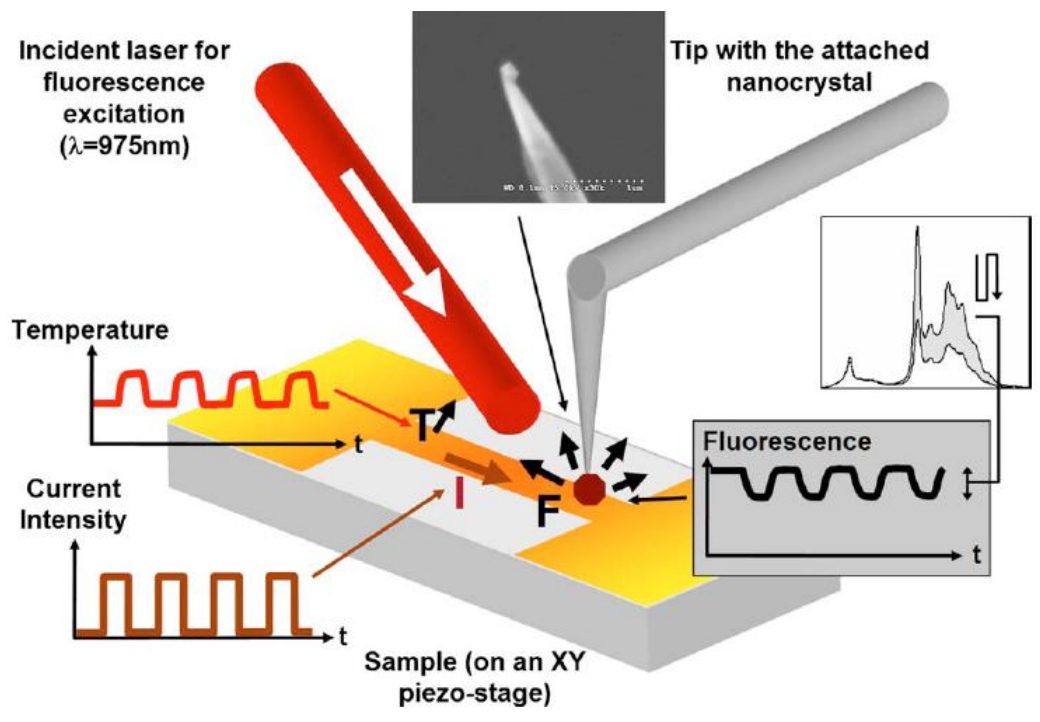


Figure 2

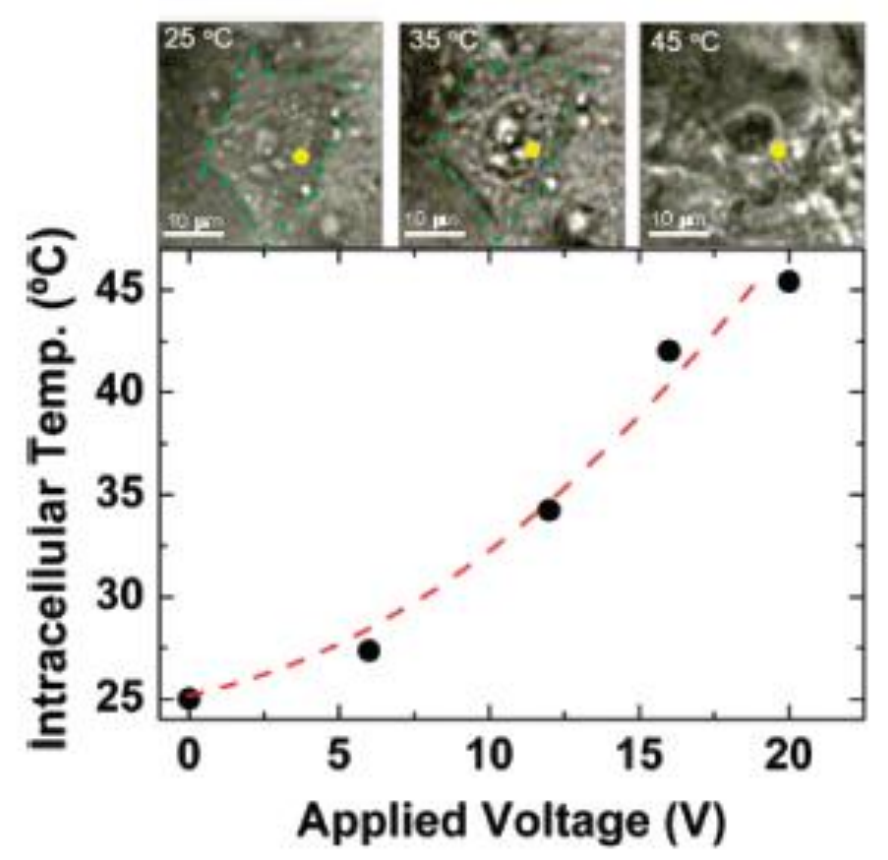


Figure 3

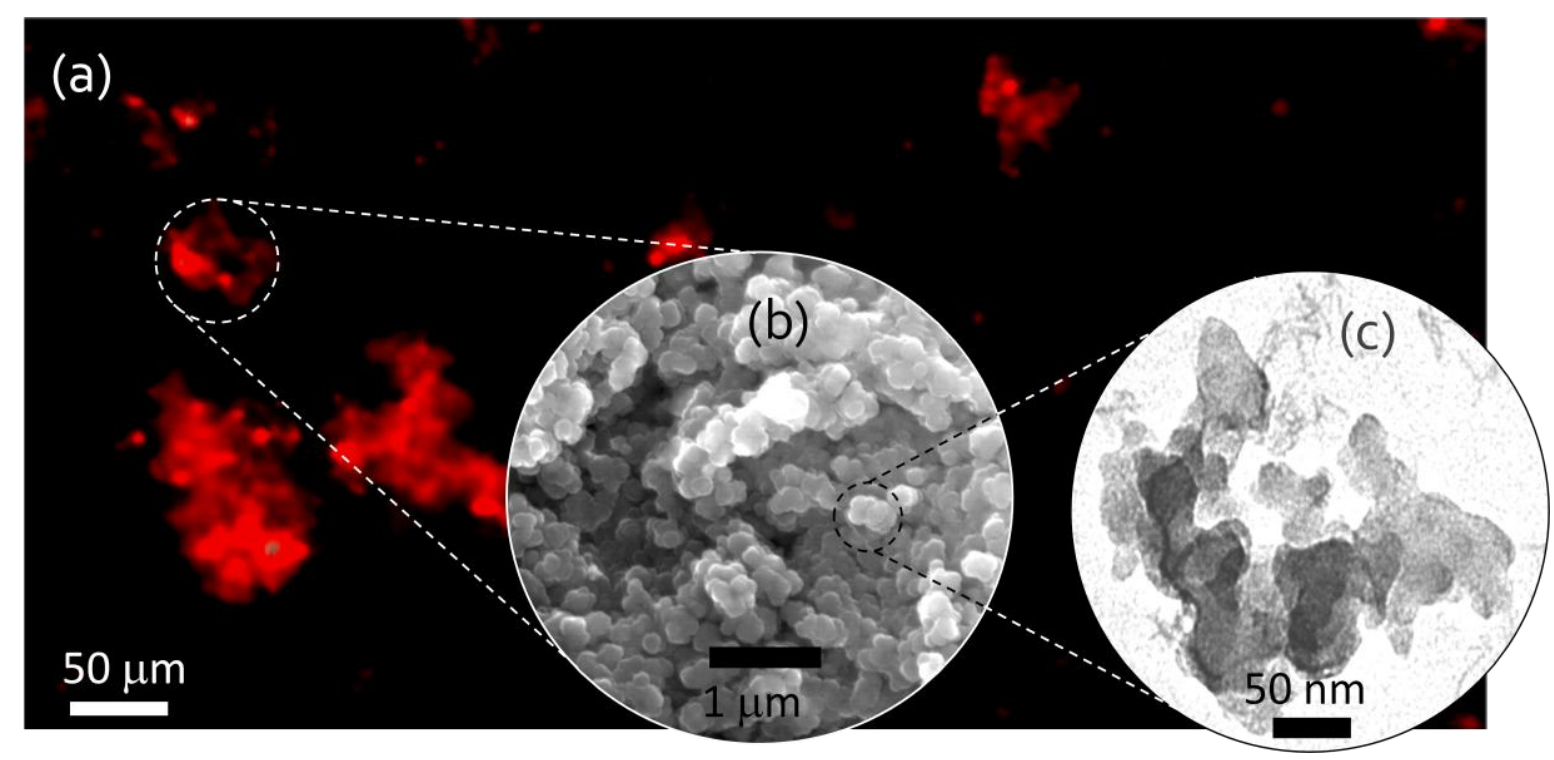


Figure 4
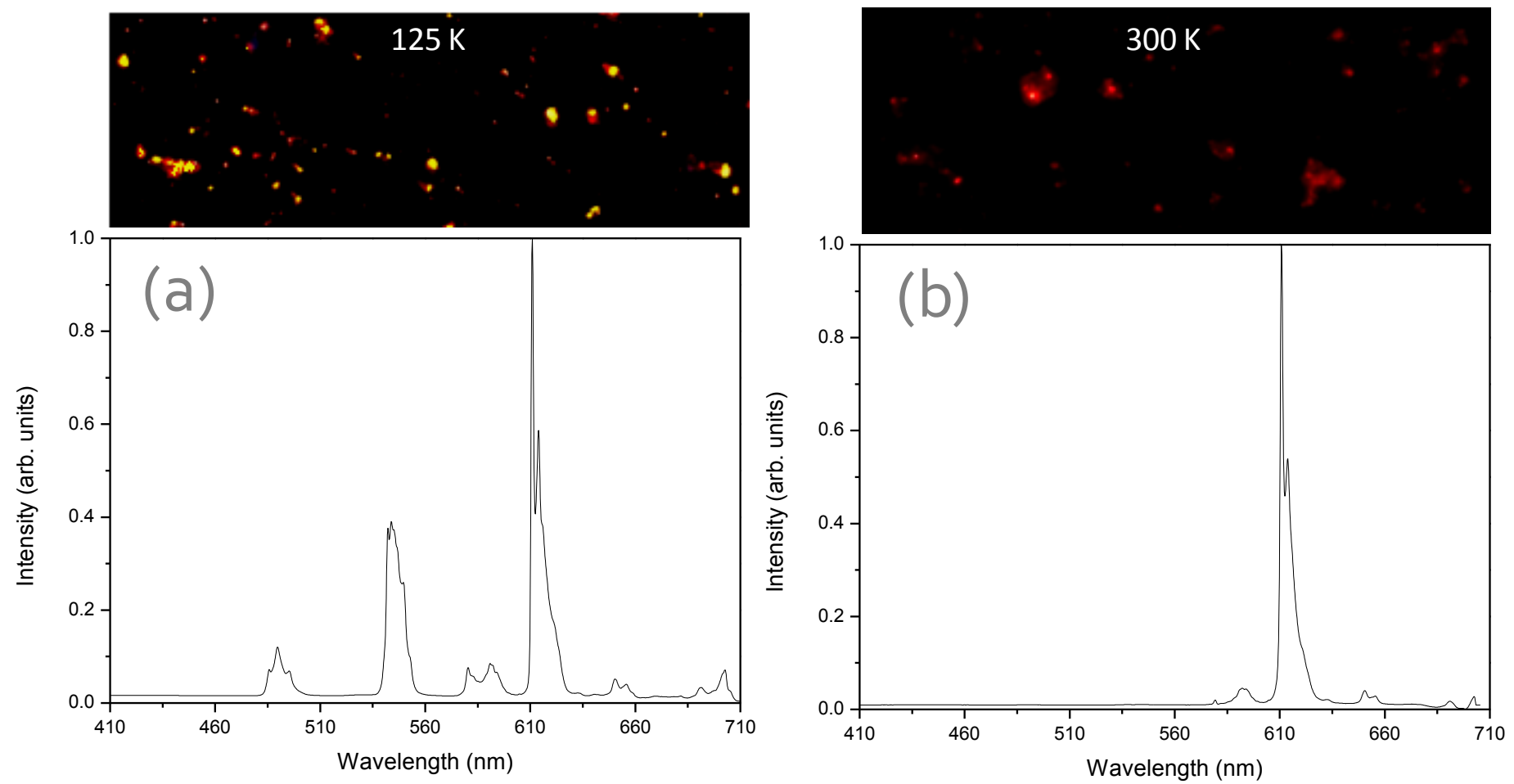
Figure 5

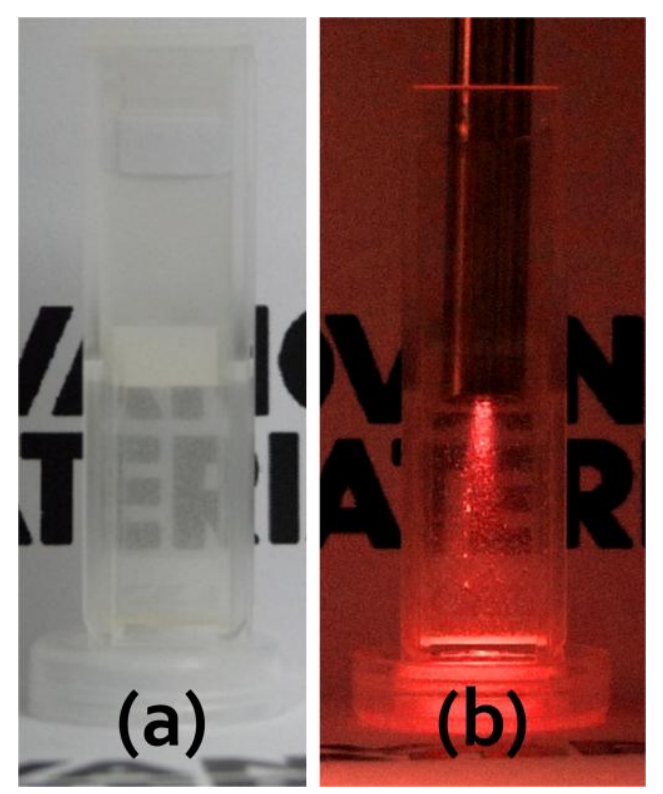

